\title{
Immunoprophylaxis of Compulsory Hepatitis B Vaccination in Sharkia, Egypt
}

\author{
Ahmad S. Sherbini ${ }^{1}$, Hanan S. Sherbiny ${ }^{2}$ \\ ${ }^{I}$ Tropical Medicine Department, Faculty of Medicine, Zagazig University, Egypt. \\ ${ }^{2}$ Pediatric Department, Faculty of Medicine, Zagazig University, Egypt.
}

Corresponding Author Ahmad S. Sherbini

Email:
ahmadsakr65@yahoo.
com

Key words: Vaccine Hepatitis $B$

Immunoprophylaxis Anamnestic reaction.
Background and study aim: Hepatitis B vaccination has been included in routine infant's immunization in Egypt since 1991. Although it could provide high levels of sero-protection, the duration of that protection remains largely unknown. The aim of this study was to evaluate the initial immunoprophylaxis response to compulsory hepatitis $\mathrm{B}$ vaccination and its long term protection in Egyptian children.

Patients and methods: Antibodies to hepatitis B surface antigen (anti-HBs) was assessed in 200 apparently healthy Egyptian children.They were all vaccinated with hepatitis $B$ vaccine in infancy through the compulsory vaccination program. They were classified into two groups, each contained 100 children. The 1 st group included infants at least 2 months after completion of the 3 doses of the vaccine ( $\geq 8$ months old). While the 2nd group included children aged 6-12 years old. Sera were obtained from all children and were tested for anti-HBs titer, $\mathrm{HBsAg}$ and antibodies to hepatitis B core antigen (anti-HBc) by ELISA. Children who were discovered to have non-protective titers $(\leq 10 \mathrm{mIU} / \mathrm{mL})$ received a booster dose of the vaccine and were re-evaluated within 2-4 weeks. Different factors associated with poor immune response were analyzed.
Results: The initial responders rate of $83 \%$ was detected in group I which was significantly higher than that in group $\Pi$ of only $52 \%$. Moreover, mean titer of Anti-Hbs was significantly decreased with age, $(48.9 \pm 15.8 \mathrm{mIU} / \mathrm{ml}$ in group I vs $12.6 \pm 5.2 \mathrm{mIU} / \mathrm{ml}$ in group II). Also, within the 2nd group, a negative correlation between Anti-HBs mean level and age in years was displayed. No significant difference could be detected in the percentage of infected children between both groups (4\% in group I vs. $6 \%$ in group II). Sixty two percent of the boosted children showed a significant increase in their mean anti-HBs titer. Male gender and rural residence were associated with poor vaccine response in both groups.

Conclusion: As maternal screening is not feasible in our country, Hep-B vaccine birth dose is a necessity to prevent perinatal infection. Most of infants vaccinated with compulsory Hep-B vaccine retained their immune protection years after vaccination either by high antiHBs titer or efficient memory $\mathrm{T}$ cells. However, large scale and long term follow up study is still needed before clearly answering the question about the need for booster dosing and its proper timing.

\section{INTRODUCTION}

Hepatitis B virus (HBV) infection is a serious public health problem worldwide and a major cause of chronic hepatitis, cirrhosis, and hepatocellular carcinoma (HCC) [1]. At least two billion people, or one third of the world's population, have been infected with HBV and an estimated 1 million people die each year from acute and chronic squeals secondary to HBV infection [2]. In addition, more than 400 million people, or $6 \%$ of the world's population, are chronic carriers of
HBV. Approximately 4.5 million new cases of HBV infection occur worldwide annually, and one fourth of these cases progress to liver disease [3].

There is a marked difference in the geographic distribution of carrier rates, ranging from $10-20 \%$ in SouthEast Asia and Sub-Saharan Africa to less than $1 \%$ in Northern America and most of Europe [1, 4].In areas with low endemicity, most HBV infections are acquired via horizontal transmission among adolescents and young adults. Conversely, in areas of 
high endemicity, the most common route of transmission is perinatal and the infection is often acquired during preschool years. The risk of becoming a HBV carrier is $90 \%$ in cases of perinatal infection, $25-30 \%$ for infected infants and children under 5 years of age, and less than $10 \%$ for infected adults $[2,4]$. More than $25 \%$ of infants and older children who acquire $\mathrm{HBV}$ infection will eventually develop HBV-related cirrhosis and/or HCC. Adults who have had chronic HBV infection since childhood develop $\mathrm{HCC}$ at a rate of $5 \%$ per decade, which is 100 to 300 times the rate among uninfected persons [1, $4]$.

In the early nineties, the prevalence of hepatitis $\mathrm{B}$ virus (HBV) carriage in Egypt was reported as high as 20\%.Considering that, most HBV infections in Egypt occurred vertically, universal infant vaccination is considered to be the key towards elimination of HBV infection [5].

The first HB vaccine, derived from human carriers' plasma, was approved for use in the United States in 1981. In 1991, the WHO recommended that all countries implement a policy of universal HB vaccination by 1997 [6]. Most countries have incorporated universal HBV vaccination into their national infant immunization programs. The estimated global coverage rate of infant $\mathrm{HB}$ vaccination increased from less than $1 \%$ in 1990 to $30 \%$ in 2000 , and from nearly $50 \%$ in 2004 to $69 \%$ in 2008 [7, 8]. This program reduced not only the rate of persistent infection and the total prevalence of $\mathrm{HBV}$ in the younger generation, but also the occurrence of childhood HCC and fulminant hepatitis [9].

The HB vaccination at $(0,1$ and 2 months or at 0 , 1 and 6 months) after birth has been compulsory for all neonates born in areas of high endemicity to prevent perinatal transmission. Infants born to HBsAg-positive mothers and mothers of unknown HBsAg status should receive the HB vaccine and hepatitis B immunoglobulin (HBIG) within 24 hours of birth. Five to twenty\% of infants born to $\mathrm{HBs} A g$-positive and $\mathrm{HBeAg}$ negative mothers and seventy to ninety\% of infants born to $\mathrm{HBeAg}$-positive mothers will get infected if not given immunoprophylaxis [3]. A compulsory vaccination program against hepatitis B infection was started in Egypt in 1991 using a yeast recombinant DNA vaccine $(10 \mu \mathrm{g})$, with a schedule of 2, 4 and 6 months of age [10]. Sero-protection is assured when hepatitis B surface antibody (anti-HBs) levels are $\geq 10$ $\mathrm{mIU} / \mathrm{mL}[11]$. Hepatitis B infection has significantly declined in the past decade as a result of $\mathrm{HB}$ vaccination and the introduction of other public health measures, such as the use of universal precautions in medical settings and blood screening tests. Recently, Egypt is considered to be a region of intermediate prevalence for HBV infection with a reported infection rate of 2-8\% [12].

The immunogenicity of hepatitis $\mathrm{B}$ vaccine has been a subject of intense research since HB vaccine was adopted in most areas of the world [13]. Although high levels of sero-protection rates provided by $\mathrm{HB}$ vaccine (both early plasma-derived and the current recombinant) have been adequately confirmed [14], recent studies have found that after neonatal immunization with $\mathrm{HB}$ vaccine, a large proportion of the children, especially adolescents, exhibited waning immunity. Such decreased protection poses the risk of breakthrough infections [15]. So, more is needed to be learned about the duration of protection and indications for booster dosing [16].

The aim of the present study is to assess immune response to hepatitis B vaccine among Egyptian children vaccinated under the compulsory vaccination program, determine factors affecting immunoprophylaxis, and evaluate the need for a birth dose and boosters.

\section{PATIENTS AND METHODS}

Participants: This prospective comparative study was performed at Pediatric and Tropical Medicine Departments, Zagazig University Hospitals, (between December 2011 and June 2013). Two hundred apparently healthy children, who came for follow up after minor illness, were enrolled in our research study. Parents were interviewed and informed about the idea of the study. Consent and two contact numbers were taken for recall and follow up as needed.

Inclusion Criteria: All participants fulfilled the following inclusion criteria: 1-.Fully vaccinated in infancy through compulsory vaccination program with three doses of recombinant hepatitis B vaccine as intramuscular injection on 2,4,6 months of age in conjunction with DPT and Polio vaccines. 2- Their gestational ages were $\geq$ 37 weeks and birth weights were $\geq 2 \mathrm{~kg}$. 3 - No history of clinically apparent acute or chronic 
hepatic illness either for the participants or their mothers.

Study Design: The children were classified into two groups according to age, Group I: included 100 infants at least two months after completion of the vaccine series ( $\geq 8$ months). They were classified according to their anti-HBs titer into: responders (anti-HBs titer was > $10 \mathrm{mIU} / \mathrm{mL}$ ), and non- responders (anti-HBs titer $\leq$ $10 \mathrm{mIU} / \mathrm{mL}$ ). Group II: included 100 children aged from 6-12 years old who received the same vaccination series as infants. They were classified according to their anti-HBs titer into: immune (who retained anti-HBs titer of > $10 \mathrm{mIU} / \mathrm{mL}$ ) and non-immune (anti-HBs titer was $\leq 10 \mathrm{mIU} / \mathrm{mL}$ ). Moreover, HBsAg was tested in all participating children. Non-immune children were boosted and re-tested after 2-4 weeks to evaluate their memory $\mathrm{T}$ cell function. Anamnestic reaction to $\mathrm{HB}$ vaccine booster was defined as: non immune subject who was yielding anti-HBs titer of $\geq 100 \mathrm{mIU} / \mathrm{mL}$ or at least four-folds rise in the anti-HBs titer within 4 weeks after booster immunization [16].

Methods: All participants were subjected to full medical history and thorough clinical examination, complete blood count, liver function tests, quantitative anti-Hbs and qualitative HBsAg \& anti-HBc titers by enzyme linked immunosorbent assay (Immlite 2000) in accordance with the manufacturer's instructions. Anti-HBc positive participants, which is considered an indicator of infection, were referred to the hepatology unit for thorough clinical evaluation and detailed investigations of their HBV markers (HBs Ag, anti-HBc, antiHBs, HBeAg and HBV-DNA by PCR). Moreover, their maternal ( $\mathrm{HBsAg}, \mathrm{HBeAg}$ as well as HBV-DNA viral load) status were also evaluated.

Statistical Methods: Our data were collected and presented using SPSS for Windows (ver.10). Descriptive values for continuous variables were given as the mean $\pm \mathrm{SD}$, while categorical variables were expressed as numbers and percentages. Student $t$ test was used to compare different study groups, Spearman correlation test was performed for the correlation analysis. $\mathrm{P}$ value of $<0.05$ was considered statistically significant.

\section{RESULTS}

To recruit 100 children in group I, 123 infants were interviewed with their parents. Twenty three infants were excluded from the study due to incompletion of vaccine series at the time of interview. The vaccine completion rate was $81 \%$. Mean ages in group I were $8.9 \pm 2.15$ months ranged from 8-12 months. Moreover, 117 children were interviewed to enroll 100 children in group II, 17 children were excluded due to unknown or incomplete hepatitis B vaccine doses during infancy. Their vaccine completion rate was $85 \%$. Mean ages in group II were $7.8 \pm 1.9$ years ranged from 6-12 years. Apart from age, no statistically significant differences in vaccine completion rates, gender, or residency were found between the two groups.

$83 \%$ of participants in group I showed protective level of anti-HBs antibodies compared to only $52 \%$ of children in group II. This data confirms a statistically significant reduction in immuneprotective rates with age (Figure 1). High statistically significant reduction in mean titers of anti-HBs occurred years after vaccination (group II), when compared to those in group I as shown in Table 1. Moreover, significant negative correlation was observed between anti-HBs quantitative level and different ages (in years) in group II (Figure 2).

No statistically significant difference in percentage of infection (expressed by ani-HBc positivity) was detected between the two studied groups (4\% vs $6 \%$ with $\mathrm{p}$ value> 0.05 ). Also positive rates of HBsAg showed no statistically significant difference between the two groups (calculated as $3 \%$ and 2\%) in group I and II respectively. We evaluated the hepatitis markers in the infected subpopulation of both groups, and investigated their maternal $\mathrm{HBsAg}, \mathrm{HBeAg} \&$ HBV-DNA status. It was observed that $3 / 4$ of infected participants in group I were positive for $\mathrm{HBs} \mathrm{Ag}$, and 2/4 of their mothers were $\mathrm{HBsAg}$ +ve but - veHBeAg with low viral loads. While in group II, 2/6 of infected children were positive for $\mathrm{HBsAg}$ and had an associated positive maternal HBsAg but -veHBeAg with low viral loads also (data not shown). 
After exclusion of the infected cases ( 2 boys and 2 girls in group I\& 4 boys and 2 girls in group II), a significant difference in the rates of antiHBs between males and females in group I with a better response to vaccine in females than males (92\% Vs75\%) was noted. Moreover, $60 \%$ of girls in group II retained their immune protective levels years after vaccination as infants versus only $41 \%$ of boys of the same group (Table 2).

To evaluate the effect of residency on the immune response, 96 children were analyzed in group I, in which $90 \%$ of those lived in cities compared to $80 \%$ from rural areas were responders to the vaccine.Furthermore,94 children were analyzed in group II where 53\% from urban residencehad retained their immune protective level of anti-HBs compared to $48 \%$ from rural residence with no statistically significant difference between the two subgroups.

Out of the non-immune46 children in group II who did not show any evidence of infection (negative for both anti-HBc and HBs antigen), only 29 returned for booster vaccination. They were retested 2-4 weeks after a single vaccine dose with mean time $23 \pm 2$ days. $62 \%$ of boosted children demonstrated significant increase in their mean anti-HBs antibody titers, almost 10 folds more than values before boosting ( $\mathrm{P}$ $<0.05)$. This anamnestic reaction is considered an indicator of efficient memory $\mathrm{T}$ cell protection against hepatitis B viral infection, 38\% did not develop such response which renders them at risk for infection upon exposure or breakthrough infections (Table 3).

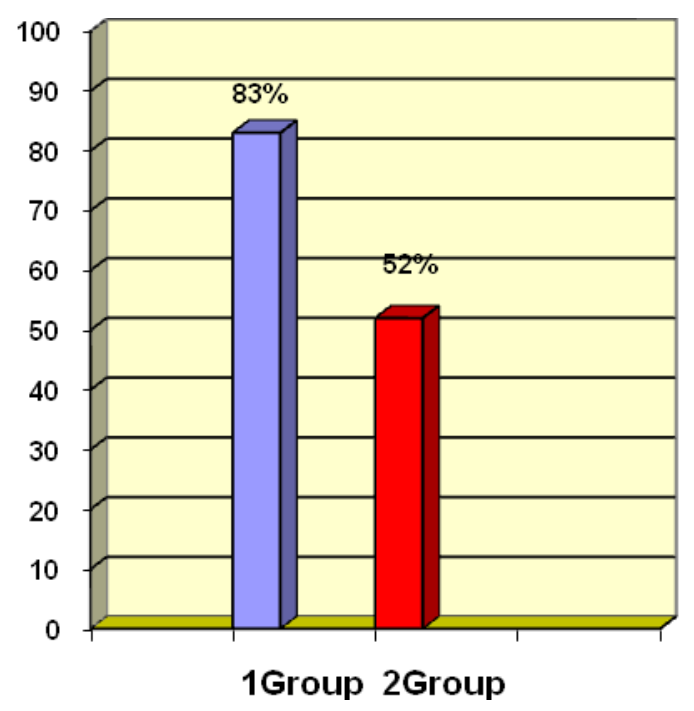

Fig. 1: Percentage of participants with protective levels of anti-HBs in studied groups

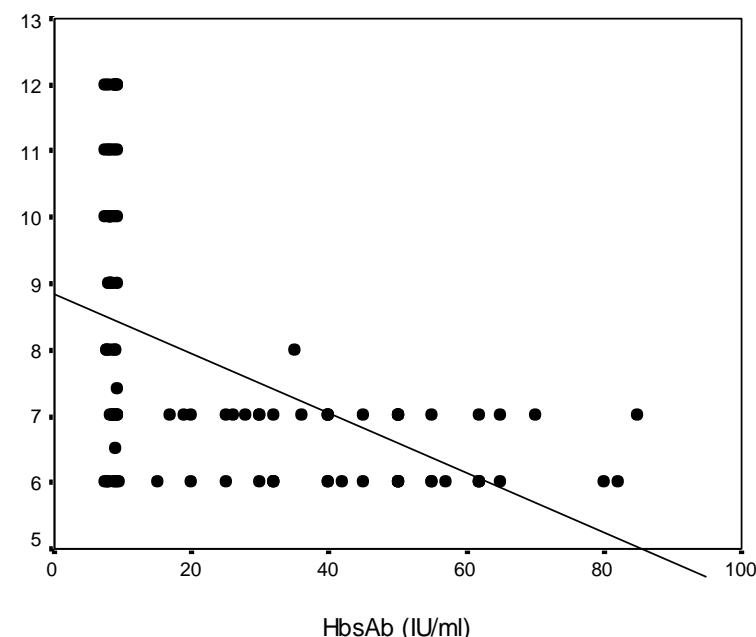

Fig. 2: Correlation between anti-HBs and different ages in group II 
Table 1: Quantitative level of anti-HBs by ELISA in studied groups

\begin{tabular}{|c|c|c|}
\hline Anti-HBs (mIU/ml) & Group I & Group II \\
\hline Mean & 48.9 & 12.6 \\
\hline S.D & 15.8 & 5.2 \\
\hline Minimum & $<10$ & 0 (undetectable) \\
\hline Maximum & 176 & 55 \\
\hline P value & \multicolumn{2}{|c|}{$<0.001 \quad$ H.S } \\
\hline
\end{tabular}

Table 2: Gender effect on positive rates for Anti-HBs in studied groups after exclusion of infected cases

\begin{tabular}{|l|c|c|c|c|c|c|c|c|c|c|}
\hline \multirow{2}{*}{ Gender } & \multicolumn{4}{|c|}{ Group I (No: 96) } & \multicolumn{5}{c|}{ Group II (No: 94) } \\
\cline { 2 - 11 } & responders & non-responder & total & immune & non-immune & Total \\
\hline Male & No. & $\%$ & No. & $\%$ & No. & No. & $\%$ & No. & $\%$ & 44 \\
\hline Female & 34 & 75 & 10 & 25 & 44 & 18 & 41 & 26 & 59 & \\
\hline$P$ & 48 & 92 & 4 & 8 & 52 & 30 & 60 & 20 & 40 & 50 \\
\hline
\end{tabular}

Table 3: Results of boostering experiment on non-immune children in group II (No: 29)

\begin{tabular}{|l|c|c|c|c|}
\hline Anti-Hbs & $\mathrm{N}$ & $\%$ & Mean & S.D \\
\hline Responders $^{\mathrm{a}}$ & 18 & 62 & 154 & 18.3 \\
\hline Non- responders & 11 & 38 & 32 & 2.5 \\
\hline
\end{tabular}

\section{DISCUSSION}

The WHO recommended universal vaccination against $\mathrm{HBV}$ to ultimately eliminate $\mathrm{HB}$ infection; this recommendation had been progressively implemented in 168 countries having universal immunization programs by the end of 2006[17].Expanded program on immunization (EPI) is a component of the child survival project (CSP). In 1991, CSP/EPI developed a national plan to introduce national immunization for infants against hepatitis B virus infection which is endemic in Egypt. Licensed vaccine against $\mathrm{HBV}$ is administrated at 2, 4, 6 months of age coinciding with oral polio vaccine (OPV) and Diphtheria, Pertussis, Tetanus (DPT). It is given either separately or in a combination form as (DPT-Hep B vaccine [18]. The present study appreciates such schedule as the vaccine completion rates are high (82\%-85\%) in our groups with no significant difference at different age groups. No extra visits are needed to receive the HepB vaccine alone, same types of vaccines are given each visit, use of combination form (DPT-Hep B) vaccine will decrease the number of injection sites, number of needles and syringes used, and also the amount of space required for cold chain storage and transport [19] .All these advantages make this schedule the easiest, to be implemented and received with high completion rate. This schedule will prevent infections acquired during early childhood as well as those acquired later in life. Unfortunately, it will not prevent perinatal infections because it does not include a birth dose [19].

Two groups were studied to evaluate immunoprophylaxis yield of this schedule during childhood. The presence of protective levels of anti-HBs $(>10 \mathrm{mIU} / \mathrm{mL})$ is considered an indicator of the initial response to the vaccine in group I and also give a clue about the immune protection level years after vaccination in group II. The significant decrease in percentage and mean values of protective levels of Anti-HBs in childhood (group II) in comparison with infants 
(group I) imply that, the majority of infants respond initially to the vaccine; but many of them lose their protective immune level during childhood. This result raises concerns about the durability of immune-prophylaxis in later childhood if the vaccine was given only in infancy.

Many follow-up studies of HB vaccine efficacy were reviewed and a wide fluctuation in their results was detected. West and his team screened children at 12 years of age who had received their $\mathrm{HB}$ vaccine in infancy and were low-risk for hepatitis B virus exposure. None of the children had anti-HBs $<10 \mathrm{mIU} / \mathrm{ml}$ with $100 \%$ retaining their immune response [20]. While, Jafarzadeh and Montazerifar,evaluated the persistence of anti-HBs in healthy Iranian children receiving primary Hep $\mathrm{B}$ vaccination at $0,1.5 \& 9$ months of age. Only $47.9 \%$ had a protective level of anti-HBs at 10 years age [21].On 1999,El-Sawy and Mohamed evaluated the long term immunogenicity and efficacy of vaccination using the schedule of $(2,4,6$ months) in 180 children whose time lapse since last vaccination dose varied between one month and five years. High seroprotection rates (93.3\%) were elicited. None of the participants had clinical hepatitis nor $\mathrm{HBsAg}$ positivity was detected in any participant but only one had positive results for anti-HBc test [22]. The longest follow up study, up to our knowledge, was that performed by Qian et al. who followed the long term immunogenicity and seroprotection in healthy individuals $1,11 \& 23$ years after vaccination with either $10 \mu \mathrm{gm}$ or $20 \mu \mathrm{gm}$ doses of plasma derived $\mathrm{HB}$ vaccine. Around 50\% of the participants still kept anti- $\mathrm{HBs}>10 \mathrm{mIU} / \mathrm{mL}$ at the age of 23 years, no clinically apparent cases of HB infection were observed throughout the entire study period [16].A major difference between the different studies was the timing of the initial vaccination dose, as some was given at birth while others 2 months later. The delay in initial vaccination series was associated with better persistence of anti-HBs. In agreement with our finding, a recent study by Agladioglu and colleague concluded that the scheme started at the end of the second month of life yielded a significantly higher immunogenicity than that started at birth [23]. This finding was explained by Hou et al., on the bases of insufficient maturation of the immune system in neonates and the possibility that maternally derived antibodies could interfere with active immunization response [24].

Mean titers of Anti-HBs antibody described at different literatures were $77 \mathrm{mIU} / \mathrm{mL}$ in Oon and co- workers and $880 \mathrm{mIU} / \mathrm{mL}$ by Goldjarb and colleagues $[25,26]$.Our results displayed much lower mean titer values $(49 \mathrm{mIU} / \mathrm{mL})$. This diminished immune response could be attributed to the short time gap between $2^{\text {nd }} \& 3^{\text {rd }}$ doses of our schedule. Middleman and colleagues suggested that increased time between the $1^{\text {st }} \&$ $2^{\text {nd }}$ as well as between the $2^{\text {nd }} \& 3^{\text {rd }}$ doses was positively correlated with increased levels of anti-HBs mean titers [27].A number of authors reported in their studies that the concentration of anti-HBs after the third injection was dependent on the interval between the second and third doses with significantly higher levels if the duration was longer [28, 29]. Wilson and Nokes in their article set forth a mathematical model of hepatitis B antibody kinetics suggesting that immune memory depends not only on the response directly to antigen stimulation, but also on other cells that continue to clone even after the antigen is out of circulation, the accumulation may continue for months after the initial priming of the immune system. Thus, the longer, the time period after priming that the clonal expansion has to continue and the larger the boosted response till another dose could be [30]. This theory reasonably explains our low anti- HBs titers.

Despite the obvious conclusion that $\mathrm{HB}$ vaccine induced protective antibody levels were gradually fading by age, the positive rates for anti-HBc and HBsAg in our study were not significantly increased at different ages.4\% versus $6 \%$ for anti-HBc and $3 \%$ versus $2 \%$ for HBsAg in groups 1 and group II respectively. Similar results were demonstrated in a report on long-term protection in a population of Alaska Natives where none of the children at age of 10 years who had received the $\mathrm{HB}$ vaccine at birth was HbsAg + ve despite fading of their anti-HBs with age, compared to $16 \%$ of participants of those $>10$ years old who were not vaccinated [31]. This could be explained by the persistence of immunologic memory. When those children were exposed to natural infection, they could quickly re-develop enough protective antibodies to combat active infection and chronic carriage [17]. 
Our booster experiment results confirmed the previous recommendation that administration of booster doses should not be based only on the level of anti-HBs but also on the measure of memory $\mathrm{T}$ cells function [32]. In the present study, $62 \%$ of children with negative anti-HBs who were challenged by booster vaccination developed an anamnestic response with significant increase in their anti-HBs mean titer to $154 \pm 18.3$ which is highly protective against infection. In another study, the vast majority of participants $(82 \%)$ developed a rapid and robust anamnestic response after a booster dose at year 23 after the primary vaccination series [16].

However, in this study 38\% of the boosted children did not experience such reaction within the same duration. Petersen and co-workers in their booster experiment found that $25 \%$ of their low-risk vaccinees, long-term immunologic memory to hepatitis $\mathrm{B}$ virus may be lost [33]. Despite of that, booster doses of HB vaccine are not currently recommended [29, 34]. Depending on the fact that, even with declining anti-HBs protective level with time, immune memory remains intact in most individuals. But with loosing of such memory protection, children are at greater risk of acquiring infection upon exposure to HBV infection as they approach adolescence and booster dose for that subpopulation is a demand! Could the long incubation period of 4-8 weeks for hepatitis B virus allow time for immune cells to prevent acute illness or chronic carriage among previous anamnestic memory loser subgroup?, a question which cannot be answered properly, except after large number and long-term follow-up studies for those sub-group into adolescence and early adulthood to detect any evidence of clinically significant break-through infection.

Some countries have chosen not to implement immunization at birth, but instead screen pregnant women for HBsAg. Hep vaccine birth dose and $\mathrm{HB}$ immunoglobulin were given only to infants born to HBsAg positive mothers [35].

Four of children in group I, were anti $\mathrm{HBc}+\mathrm{ve}$, of them 3/4 were +ve HBs Ag, 2/4 had associated +ve maternal $\mathrm{HBsAg}$ as well, indicating that perinatal transmission is a possibility. Lack of regular antenatal care facilities in our community, where maternal HBsAg status cannot be determined, makes first dose at birth for all infants a mandate to prevent perinatal transmission. The rationale for this suggestion is supported by the very high probability of developing chronic carrier state if the infection is acquired perinatally [36]. 20-90\% incidence of chronic carrier occurs if infection was acquired $<5$ years, perinatal infection is an important source during this period [37].Same recommendation was recently advised depending on the fact that, as maternal screening is costly and not usually feasible in developing countries so vaccination at birth is still a necessity [16].

It is important to determine predictors of nonresponsiveness to hepatitis $\mathrm{B}$ vaccine and also to evaluate risk factors of losing the sero-protection status with age. The percentages of responsiveness in females were higher than males in both study groups. Similar results were observed in adult volunteers [38]. Dentico and colleagues also reported that male gender was considered one of the risk factors associated with non-response to $\mathrm{HB}$ vaccine [39]. In contrast, Jafarzadeh and Montazerifar reported equivalent seroprotection rates and mean titers of Anti-Hbs in both genders [21]. A trend toward higher responders' rate and higher sero-protection rate were observed among urban residents when compared to rural residents. These results can be explained by the common practice that cities usually have increased number of health care centers, better quality control, better supervision, more trained personnel and more supply of equipment for cold chain storage and transport. All previous advantages guaranteed perfect vaccine implementation and so subsequent better results even for the same vaccine brand given to the same population.

\section{RECOMMENDATIONS}

The hepatitis $\mathrm{B}$ vaccine of $0,2,9$ months schedule is suggested to be followed in our country. As this regimen will prevent perinatal infection, through its birth dose for all live births; stimulate higher anti-Hbs response by wider time gap between $2^{\text {nd }}, 3^{\text {rd }}$ doses. While still coincide with already present vaccines' schedules but different vaccines are to be given at $2^{\text {nd }}$ (DPT, polio, Hep.B) and $3^{\text {rd }}$ (hepatitis $\mathrm{B}$, measles) doses.

No need for booster doses until the end of childhood and early adolescence as the majority of vaccinees are protected either by keeping their sero-protective level at this age or through developing higher levels of anti-HBs 
(anamnestic response) if exposed to the virus $\mathrm{Ag}$ through their immunologic memory stimulation. However, large-scale and longer follow-up study to evaluate memory anamnestic loser's outcome is needed to safely answer the question; do we need booster or absolutely not?

Funding: Non.

Conflicts of interest: The authors declare no conflict of interest.

Ethical approval:The Study was approved by the Ethical Committee of Faculty of Medicine , Zagazig Universty. Informed consents were obtained from the parents.

\section{REFERENCES}

1. Cha C, Dematteo RP . Molecular mechanisms in hepatocellular carcinoma development. Best Pract Res ClinGastroenterol 2005; 19: 25-37.

2. Ganem D, Prince AM, . Hepatitis B virus infection: natural history and clinical consequences. $N$ Engl J Med 2004; 350: 1118-1129.

3. Goldstein ST, Zhou F, Hadler SC, Bell BP, Mast EE, et al.A mathematical model to estimate global hepatitis B disease burden and vaccination impact.Int J Epidemiol 2005; 34: 1329-1339.

4. Lok AS.Prevention of hepatitis B virusrelated hepatocellular carcinoma.Gastroenterology $\quad 2004 ; 127$ :S303-S309.

5. Toukan A.The Middle East Regional Study Group: Strategy for the control of hepatitis B viral infection in middle east and north Africa. Vaccine 1990;8:117-21.

6. World Health Organization.Expanded programme on immunization. Global Advisory Group. Weekly Epidemiol Rec 1992; 3: 11-16.

7. World Health Organization.Statistics on hepatitis B. www.Who.Int/immunization monitoring-disease-hep_ coverage.jpg.

8. World Health Organization. Vaccines and biologicals: WHO vaccine preventable disease monitoring system: global summary 2009 (data up to 2008). www.who.int/immunization_monitoring /diseases/HepB_map_schedule.jpg.
9. Chen DS. Hepatitis B vaccination: the key towards elimination and eradication of hepatitis B. J Hepatol 2009; 50: 805-816.

10. FloreaniA ,Bordly WC, Clark SJ . Long-term persistence of anti-HBs after vaccination against HBV: an 18-year experience in health care workers. Vaccine, 2004; 22(56):607-610.

11. Yu AS, Cheung RC, Keeffe EB.Hepatitis B vaccines. Clinics in liver disease, 2004; 8(2):283-300.

12. El-Zayadi A.: Hepatitis B Virus Infection the Egyptian Situation. Arab J Gastroenterol.2007; 8:94- 98.. .

13. Lu CY, Ni YH, Chiang BL, Chen PJ, Chang $\mathrm{MH}$, et al. Humoral and cellular immune responses to a hepatitis $\mathrm{B}$ vaccine booster 15-18 years after neonatal immunization. $J$ Infect Dis 2008; 197:1419-26; PMID:18444799; http://dx.doi.org/10.1086/587695.

14. Zanetti AR, Van Damme P, Shouval D. The global impact of vaccination against hepatitis B: a historical overview. Vaccine 2008; 26:6266-73; $\quad$ PMID:18848855; http://dx.doi.org/10.1016/j.vaccine.2008.09.0 56.

15. 15-Bialek SR, Bower WA, Novak R, Helgenberger L Auerbach SB, Williams IT, Bell BP . Persistence of protection against hepatitis $\mathrm{B}$ virus infection among adolescents vaccinated with recombinant hepatitis B vaccine beginning at birth: a 15year follow-up study. Pediatr Infect Dis $J$ 2008;27:881-5.

16. Qian WU, GuiHZ ,Hou TG , ShanDP etal.,: Comparison of long-term immunogenicity $(23$ years) of $10 \mu$ and $20 \mu$ doses of hepatitis B vaccine in healthy children. Human Vaccines \&Immunotherapeutics2012; 8:1071-75.

17. Herk VK, Van Damme P: benefits of early hepatitis B immunization programs for newborns and infants. Pediatr Infect Dis J. 2008; 27: 861-9.

18. Mansour E, Abdul-Rahim S, Batouty G, Zaghloul I, Abdel-Hadi S. Integration of hepatitis B immunization in the Expanded Program on Immunization of the Child Survival Project. J Egypt Public Health Assoc. 1993; 68(5-6):487-94. 
19. World Health Organization. Hepatitis B: Fact Sheet 204.World Health Organization; [2000].WHO Web site. 2000. http://who.int/inf-fs/en/fact204.html

20. West DJ, Calandra GB. Vaccine induced immunologic memory for hepatitis B surface antigen: implications for policy on booster vaccination. Vaccine. 1996;14:1019-1027.

21. Jafarzadeh A, Montazerifar SJ. Persistence of anti-HBs antibody and immunological memory in children vaccinated with hepatitis B vaccine at birth. $J$ Ayub Med Coll Abbottabad.2006 ; 18: 4-9.

22. El-Sawy IH, Mohamed ON.Long-term immunogenicity and efficacy of a recombinant hepatitis B vaccine in Egyptian children.East Mediterr Health J. 1999 Sep; 5(5):922-32.

23. Agladioglu S, Beyazova U, Camurdan D. Immunogenicity of recombinant hepatitis $b$ vaccine : comparison of two different vaccination schedules. Infection 2010; 38:269-273.

24. Hou $\mathrm{H}$, Lingling $\mathrm{W}$, Zhang J, Zhou J. Identification of risk factors associated with immunoprophylaxis failure to prevent vertical transmission of hepatitis B virus. Journal of Infection 2013;66:447-452.

25. Oon CJ, Tank L, Gohk T et al . Evolution of a low dose Hepatitis $\mathrm{B}$ vaccine given within a childhood immunization program in Singapore.J Infect 1986, 13:255 - 267.

26. Goldfard J, Medendop S, Garcia H , Magamori $\mathrm{K}$ et al. Comparison study of the immunogenicity and safety of 5 and $10 \mu$ dosage of recombinant hepatitis $B$ vaccine in healthy infant. PedInf Dis J 1996; 764 767.

27. Middleman AB, Clandia A, Konzinetz M. The effect of late doses on the achievement of Sero protection and Anti-body titer levels with hepatitis B Immunization among Adolescents. Pediatrics 2001; 107 (5):10651069.

28. Lu CY 1. Waning immunity to plasmaderived hepatitis B vaccine and the need for boosters 15 years after neonatal vaccination. Hepatology, 2004 ;40(6):14151420 .
29. GirishaKM ,Kamat JR, Nataraj G. Immunological response to two hepatitis B vaccines administered in two Different schedules. Indian J.of pediatrics2006;73(6) : 489-49.

30. Wilson JN, Nokes DJ. Do we need 3 doses of hepatitis B vaccine? Vaccine 1999; 16: $2667-2673$.

31. Harpaz R, McMahon BJ, Margolis HS, et al: Elimination of new chronic hepatitis B infections: Results of the Alaska immunization program. J Infect Dis. 2000; 181:413-8.

32. Hadler SC: Are booster doses of hepatitis B vaccine necessary. Ann Intern Med, 1988; 108: 457 - 458 .

33. Petersen KM, Bulkow LK, Brain $\mathrm{J}$ et al. Duration of hepatitis B immunity in low risk children receiving Hepatitis B vaccination from birth.Pediatr Infect Dis $J$ 2004; 23(7): 650-655

34. Centers for Disease Control and Prevention (CDC): Acute hepatitis B among children and adolescents: United States, 1990-2002. MMWR Morb Mortal Wkly Rep. 2004

35. EUROHEP.NET. Recommendations for hepatitis B prevention strategies. Available at: ww.eurohep.net. Accessed 22 March 2010 .

36. Chen CJ, Wang LY, Yu MW. Epidemiology of hepatitis B virus infection in the AsiaPacific region. J GastroenterolHepatol2000; 15:E3-6.

37. Mahony F.J. Update on diagnosis, Management and prevention of hepatitis B virus Infection. Clinical Microbiology, 1999: $351-366$.

38. Hussein Z, Aliss, Raish $\mathrm{M}$ et al. Evolution of immunogenicity produced in India. World $J$ Gastroenterol 2005. 11(45): 7165 - 7168.

39. Dentico P, Buongiorro R, Volpe A et al. Long term immunogenicity safety and efficacy of a recombination hepatitis B vaccine in healthy adult. Eur. J Epidemol 1992; 8: $650-655$.

Peer reviewer: Amira Suliman;Professor of Tropical Medicine,Zagazig University,Egypt.Editor: Tarik Zaher; Professor of Tropical Medicine , Zagazig University,Egypt. 